Matematikai Közlemények

IV. kötet, 2016

doi:10.20312/dim.2016.01

\title{
On Friedrichs-Velte and related constants of the union of overlapping domains
}

\author{
Sándor Zsuppán \\ Berzsenyi Dániel Evangélikus (Líceum) Gimnázium és Kollégium \\ zsuppans@gmail.com
}

\begin{abstract}
ÖSSZEFOGLALÓ. Átfedő síkbeli és térbeli tartományok egyesítésének FriedrichsVelte konstansára adunk felső becslést a résztartományok megfelelő konstansai és mérete segítségével. Az eredményt összehasonlítjuk már ismert, a Babuška-Aziz és Korn konstansokra vonatkozó hasonló felső becslésekkel.
\end{abstract}

\begin{abstract}
An upper estimation for the Friedrichs-Velte and related constants of the union of overlapping planar and spatial domains is given in terms of the constants and sizes of the parts and of the size of their intersection. The estimation is compared to similar results for the same and for the related Babuška-Aziz and Korn constants.
\end{abstract}

\section{Introduction}

Motivated by problems in planar linear elasticity Friedrichs [7] introduced an inequality between the norms of two square integrable conjugate harmonic functions defined on a plane domain provided one of the functions fulfils a certain side condition and the boundary of the problem domain is piecewise smooth. His inequality and the domain specific optimal constant figuring therein are connected with some other important inequalities and corresponding constants such as the Babuška-Aziz inequality for the divergence equation, the inf-sup condition in the context of Navier-Stokes flows and for some smooth classes of domains also Korn's second inequality in linear elasticity, [2,3,9]. Friedrichs inequality remains valid for more general domains and it was generalized for other spaces of harmonic functions, [2,6,12].

In [15] Velte proved two analogous inequalities for three-dimensional simply connected domains having certain boundary regularity. He also investigated the connection of these inequalities and of the corresponding optimal constants with the Babuška-Aziz inequality, with the inf-sup condition and with the Cosserat spectrum of the domain.

For the numerical utilization of the constants we refer to $[10,13,14]$.

Despite of their importance exact values of all these constants are known in a very few cases. A useful upper estimation of Friedrichs constant for the class of star-shaped planar domains given first in [9] has been revised recently in [2,3]. Reference [11] contains an analogous upper estimation for the Velte constant of a spatial star-shaped domain.

The aim of the present paper is to give an upper estimation for the Friedrichs and Velte constants of the union of two overlapping domains. The estimation is derived essentially in the same way for two and three-dimensional domains as well. It was motivated by similar estimations for the related Babuška-Aziz and Korn constants.

In Section 2 we explain the notation and we recall some preliminary results concerning the Friedrichs-Velte and related constants. 
In Section 3 we derive the main result and compare it to corresponding known ones from $[2,5,8,10,11]$.

\section{Friedrichs-Velte and related constants}

Let $\Omega$ be a bounded planar or spatial domain the boundary $\partial \Omega$ of which will be further specified below. We denote by $|\Omega|$ the size of $\Omega$ which means the area for plane domains and the volume for spatial domains. Let $L_{2}(\Omega)$ be the usual Hilbert space of square integrable functions over $\Omega$. For $f, g \in L_{2}(\Omega)$ we denote by $\langle f, g\rangle_{0, \Omega}=\int_{\Omega} f g$ their inner product, and by $\|f\|_{0, \Omega}$ the norm of $f$. The integral mean of $f$ over $\Omega$ is $\langle f\rangle_{\Omega}=\frac{1}{|\Omega|}\langle 1, f\rangle_{0, \Omega}$. For vector valued functions $f, g \in L_{2}(\Omega)^{n}$ we use the inner product $\langle f, g\rangle_{0, \Omega}=\int_{\Omega} \sum_{k=1}^{n} f_{k} g_{k}$ and the respective norm.

The Friedrichs inequality reads in the notation of the present paper as follows.

Proposition 2.1 (Shapiro, [11]) Let $\Omega$ be a bounded plane domain satisfying an interior cone condition and let $w_{0} \in \Omega$. Let $u$ and $v$ be arbitrary square integrable conjugate harmonic functions on $\Omega$. Then, for some finite positive constants $\Gamma(\Omega)$ and $\Gamma\left(\Omega, w_{0}\right)$, which do not depend on $u$ and $v$ Friedrichs inequality holds in either of the forms

$$
\begin{gathered}
\|u\|_{0, \Omega}^{2} \leq \Gamma(\Omega)\|v\|_{0, \Omega}^{2} \text { provided }\langle u\rangle_{\Omega}=0 \text { or } \\
\|u\|_{0, \Omega}^{2} \leq \Gamma\left(\Omega, w_{0}\right)\|v\|_{0, \Omega}^{2} \text { provided } u\left(w_{0}\right)=0 .
\end{gathered}
$$

According to [2] Friedrichs inequality remains valid for the larger class of planar John domains.

The optimal constant $\Gamma(\Omega)$, called Friedrichs constant of the plane domain $\Omega$, is the least positive number such that the inequality (1) is fulfilled for all pairs of conjugate harmonic functions $u$ and $v$. The exact value of $\Gamma(\Omega)$ depends only on the shape of $\Omega$ but does not depend on its size. $\Gamma\left(\Omega, w_{0}\right)$, called Friedrichs constant with respect to the point $w_{0}$, depends additionally on the interior point $w_{0}$. We have

$$
1 \leq \Gamma(\Omega) \leq \Gamma\left(\Omega, w_{0}\right) \leq \frac{|\Omega|}{\left|D\left(w_{0}, r\right)\right|} \Gamma(\Omega) .
$$

where $D\left(w_{0}, r\right)$ denotes any disc centered in $w_{0}$ with radius $r$ contained in the interior of $\Omega$, see $[16]$.

In order to formulate a three-dimensional analogy of Friedrichs inequality Velte [15] considers harmonic functions $u$ and $v=\left(v_{1}, v_{2}, v_{3}\right)$ of three variables conjugate in the sense of the Moisil-Teodorescu equations

$$
\operatorname{rot} v=-\nabla u \text { and } \operatorname{div} v=0 .
$$

Using these notations, the Velte inequalities are the following.

Proposition 2.2 (Velte, [14]) Let $\Omega$ be a bounded simply-connected spatial domain with $C^{2}$ boundary. Then there are constants $\Gamma(\Omega) \geq 1$ and $\tilde{\Gamma}(\Omega) \geq 1$ depending only on the shape of $\Omega$ such that for any pair $u$ and $v$ conjugate in the sense of (4) the inequalities

$$
\|u\|_{0, \Omega}^{2} \leq \Gamma(\Omega)\|v\|_{0, \Omega}^{2} \text { provided }\langle u\rangle_{\Omega}=0 \text { and }
$$




$$
\|v\|_{0, \Omega}^{2} \leq \tilde{\Gamma}(\Omega)\|u\|_{0, \Omega}^{2} \text { provided } v \cdot n=0 \text { on } \partial \Omega
$$

hold, where $n$ denotes the outer unit normal to $\partial \Omega$.

The optimal constant $\Gamma(\Omega)$, called Velte constant of the spatial domain $\Omega$, is the least positive number such that the inequality (5) is fulfilled for all pairs of conjugate harmonic functions $u$ and $v$.

Obviously, there is an analogy between (1) in the planar case and (5) in the spatial case involving not only the inequalities but also the corresponding normalizations. Indeed, if we set the first two coordinates of the vector function $v$ in (4) zero and if we let the third coordinate $v_{3}$ depend only on $x_{1}$ and $x_{2}$, then (4) reduce to the Cauchy-Riemann equations between the harmonic functions $u$ and $-v_{3}$ and (5) reduces to (1).

Justified by this analogy we denote in this paper by $\Gamma(\Omega)$ both Friedrichs and Velte constants and refer to them as Friedrichs-Velte constants.

In [4] inequalities for conjugate harmonic differential forms are examined, which contain the Friedrichs and Velte inequalities as special cases.

Also in [4] the author derives also a correspondence between the Friedrichs-Velte constant and the Babuška-Aziz constant $C(\Omega)$ figuring in the Babuška-Aziz inequality, which guaranties the stable solvability of the divergence equation $\operatorname{div} u=q$ for $q \in L_{2}(\Omega)$ with $\langle q\rangle_{\Omega}=0$ in the Sobolev space $H_{0}^{1}(\Omega)^{n}$ of vector functions with square integrable gradient over $\Omega$ and zero trace on the boundary, i.e. we have $|u|_{1, \Omega} \leq C\|q\|_{0, \Omega}$ for the solution $u$ with some positive finite constant $C$ depending not $u$, where $|u|_{1, \Omega}=\|\operatorname{grad} u\|_{0, \Omega}$. The BabuškaAziz constant $C(\Omega)$ of the domain $\Omega$ is the least possible of the above constants, c.f $[1,2,4]$.

Proposition 2.3 (Costabel, $[2,4]$ ) For any bounded open set $\Omega$ the Babuška-Aziz constant $C(\Omega)$ is finite iff the Friedrichs-Velte constant $\Gamma(\Omega)$ is finite, and there holds

$$
C(\Omega)=\Gamma(\Omega)+1 \text {. }
$$

Both constants are further related to other important domain specific constants, such as the inf-sup constant $\beta(\Omega)$ and the Cosserat constant $\sigma(\Omega)$ :

$$
\Gamma(\Omega)+1=C(\Omega)=\frac{1}{\sigma(\Omega)}=\frac{1}{\beta^{2}(\Omega)^{\prime}}
$$

and if the boundary of the domain is smooth enough to the Korn constant $K(\Omega)$ of the domain $K(\Omega)=2 C(\Omega)$, c.f. $[1,2]$.

\section{Main result}

\subsection{Estimations}

Despite of their importance exact values of the Friedrichs-Velte constants are known only for a few domains. Such examples are the disc, the ellipse, some domains obtainable as conformal maps of the unit disc in the plane [17,18] and the sphere in three dimensions. However there are useful upper estimations for star-shaped domains, see [2,3,9] for planar and [11] for spatial domains. In order to obtain estimations for the examined constants of other than star-shaped domains, one can consider unions of (star-shaped) domains and derive estimations for the constants of these unions in terms of the constants of the parts, see [8]. In this section we develop such an estimate for the Friedrichs-Velte constant of the overlapping union of two arbitrary planar or spatial domains, and compare it to other existing ones for the 
Babuška-Aziz and Korn constants. In order to simplify the notation we denote the norm $\|\cdot\|_{0, \Omega}$ in this section by $\|\cdot\|$. First we need the following

Lemma 3.1 Let $\Omega$ denote a bounded planar or spatial domain, and let $A$ be a subdomain of $\Omega$ such that $|A|>0$. If $\langle f\rangle_{\mathrm{A}}=0$ for $f \in L_{2}(\Omega)$, then there follows

$$
\left\|f-\langle f\rangle_{\Omega}\right\|^{2} \leq\|f\|^{2} \leq \frac{|\Omega|}{|A|}\left\|f-\langle f\rangle_{\Omega}\right\|^{2} .
$$

PROOF. One easily verifies the equality

$$
\|f\|^{2}=\left\|f-\langle f\rangle_{\Omega}\right\|^{2}+|\Omega|\langle f\rangle_{\Omega}^{2} .
$$

Omitting the nonnegative term on the right-hand side of (10) yields the left-hand side inequality. Next we use $\langle f\rangle_{\mathrm{A}}=0$ and estimate by the Cauchy-Schwarz inequality:

$$
\left(\int_{\Omega} f\right)^{2}=\left(\int_{\Omega \backslash A} f\right)^{2} \leq|\Omega \backslash A| \cdot \int_{\Omega \backslash A} f^{2} \leq(|\Omega|-|A|) \cdot \int_{\Omega} f^{2}
$$

Substituting this into (10) and rearranging gives

$$
\frac{|A|}{|\Omega|}\|f\|^{2} \leq\left\|f-\langle f\rangle_{\Omega}\right\|^{2}
$$

which is equivalent to the right-hand side inequality in (9). Equality occurs here if we set $f=$ $1-\chi_{A}$, where $\chi_{A}$ means the characteristic function of the subdomain $A$.

The left-hand side of (9) was implicitly already utilized in [7] and [9] in the planar case and in [11] in the spatial case.

Lemma 3.1 makes it possible to change the normalization $\langle u\rangle_{\Omega}=0$ in (5) to $\langle u\rangle_{A}=0$ for some subdomain $A \subseteq \Omega$ in order to obtain a modified version of the Friedrichs-Velte inequalities.

Lemma 3.2 Let $\Omega$ be a bounded planar or spatial domain and let $A$ be a subdomain of $\Omega$ such that $|A|>0$. If the Friedrichs-Velte inequality holds on $\Omega$ with the Friedrichs-Velte constant $\Gamma(\Omega)$, then there is a constant $\Gamma(\Omega, A)$ depending only on the domain $\Omega$ and its subdomain $A$ such that the Friedrichs-Velte inequality holds for conjugate harmonic functions satisfying the normalization $\langle u\rangle_{A}=0$ instead of $\langle u\rangle_{\Omega}=0$. Moreover, the optimal constants $\Gamma(\Omega)$ and $\Gamma(\Omega, A)$ are connected by

$$
\Gamma(\Omega) \leq \Gamma(\Omega, A) \leq \frac{|\Omega|}{|A|} \Gamma(\Omega) .
$$

PROOF. Substitute the result of Lemma 3.1 into the Friedrichs-Velte inequality.

Remark. The Friedrichs-Velte constants $\Gamma(\Omega, A)$ of the domain with respect to a subdomain can be seen as a generalization of the Friedrichs-Velte constants $\Gamma\left(\Omega, w_{0}\right)$ with respect to an interior point.

The main result of this paper is the following

Theorem 3.3 Let be $\Omega=\Omega_{1} \cup \Omega_{2}$ and $\Omega_{s}=\Omega_{1} \cap \Omega_{2}$ such that $\left|\Omega_{s}\right|>0$. If the FriedrichsVelte inequality holds for either of the domains $\Omega_{1}$ and $\Omega_{2}$, then it holds also for their union $\Omega$ and there also follows

$$
\Gamma(\Omega) \leq \frac{\left|\Omega_{1}\right|}{\left|\Omega_{s}\right|} \Gamma\left(\Omega_{1}\right)+\frac{\left|\Omega_{2}\right|}{\left|\Omega_{s}\right|} \Gamma\left(\Omega_{2}\right)
$$


PROOF. We give the proof in the spatial case, but it is practically the same in the planar case. Let the conjugate pair $u, v \in L_{2}(\Omega)$ be such that $\langle u\rangle_{\Omega_{s}}=0$.

$$
\frac{\|u\|^{2}}{\|v\|^{2}}=\frac{\int_{\Omega} u^{2}}{\int_{\Omega}|v|^{2}}=\frac{\int_{\Omega_{1}} u^{2}+\int_{\Omega_{2}} u^{2}-\int_{\Omega_{S}} u^{2}}{\int_{\Omega}|v|^{2}} \leq \frac{\int_{\Omega_{1}} u^{2}}{\int_{\Omega_{1}}|v|^{2}}+\frac{\int_{\Omega_{2}} u^{2}}{\int_{\Omega_{2}}|v|^{2}}
$$

By Lemma 3.2 there follows

$$
\frac{\|u\|^{2}}{\|v\|^{2}} \leq \Gamma\left(\Omega_{1}, \Omega_{s}\right)+\Gamma\left(\Omega_{2}, \Omega_{s}\right)
$$

which implies

$$
\Gamma\left(\Omega, \Omega_{s}\right) \leq \Gamma\left(\Omega_{1}, \Omega_{s}\right)+\Gamma\left(\Omega_{2}, \Omega_{s}\right) .
$$

The estimation (14) immediatly follows by using the inequality (13).

Example 3.4 Theorem 3.3 can be utilized to obtain upper estimates for Friedrichs-Velte constants of more general domains using some known upper bounds for simpler ones. To exemplify this let $\Omega_{1}$ be an L-shaped domain which is the union of two rectangles with sides 1 and $L+1(L>1)$ which intersect in a square with sidelength 1 . The domain $\Omega_{1}$ is star-shaped and the estimation from [2] yields $\Gamma\left(\Omega_{1}\right) \leq\left(L+\frac{1}{2}+\sqrt{L^{2}+L+\frac{1}{2}}\right)^{2}$. Let $\Omega_{2}$ be congruent to $\Omega_{1}$ and set $\Omega=\Omega_{1} \cup \Omega_{2}$ such that $\Omega=\Omega_{1} \cap \Omega_{2}$ is a rectangle with sides 1 and $L+1$. For the domain $\Omega$, which is not star-shaped, Theorem 3.3 gives the upper estimation $\Gamma(\Omega) \leq$ $\left(4-\frac{2}{L+1}\right) \Gamma\left(\Omega_{1}\right)$ which differs from that for $\Gamma\left(\Omega_{1}\right)$ only by a factor at most 4 .

\subsection{Comparison}

In this section we compare the main result to existing ones for the related constants in the case of planar and spatial domains as well.

First we consider Theorem 3.1 in [8] for the Babuška-Aziz constant of the union of finitely many overlapping star-shaped domains. A direct comparison is impossible because this result does not contain the exact Babuška-Aziz constants of the subdomains but only an upper estimation of their value. In order to overcome this difficulty we follow the proof of Theorem 3.1 in [8] for the case of the union of two overlapping domains and reformulate it with keeping the Babuška-Aziz constants of the subdomains in the resulting formula. We achieve the following

Proposition 3.5 Let be $\Omega=\Omega_{1} \cup \Omega_{2}$ and $\Omega_{s}=\Omega_{1} \cap \Omega_{2}$ such that $\left|\Omega_{s}\right|>0$. If the BabuškaAziz inequality holds for either of the domains $\Omega_{1}$ and $\Omega_{2}$, then it holds also for their union $\Omega$ and there also follows

$$
C(\Omega) \leq \max _{j=1,2}\left\{\frac{\left|\Omega_{j}\right|}{\left|\Omega_{s}\right|} C\left(\Omega_{j}\right)\right\} .
$$

We utilize Corollary 3.5 only as a comparison to the main Theorem 3.3, however, it could be of interest on its own.

To this end we substitute (7) into (18) and we obtain

$$
\Gamma(\Omega) \leq \max _{j=1,2}\left\{\frac{\left|\Omega_{j}\right|}{\left|\Omega_{s}\right|} \Gamma\left(\Omega_{j}\right)+\frac{\left|\Omega_{j} \backslash \Omega_{s}\right|}{\left|\Omega_{s}\right|}\right\} .
$$


This shows that (14) gives a better upper estimation for $\Gamma(\Omega)$ than (19) if $\left|\Omega_{1} \backslash \Omega_{s}\right|>$ $\left|\Omega_{2}\right|$ and $\Gamma\left(\Omega_{2}\right) \leq \frac{\left|\Omega_{1} \backslash \Omega_{s}\right|}{\left|\Omega_{2}\right|}$.

Another possibility for comparison gives an analoguos result from [10] for the related Korn constant $K(\Omega)$ in the case of smoothly bounded planar simply-connected domains. It reads

$$
K(\Omega) \leq \max _{j=1,2}\left\{K\left(\Omega_{j}\right)+\frac{\left|\Omega_{j}\right|}{\left|\Omega_{s}\right|}\left(\sqrt{K\left(\Omega_{1}\right)}+\sqrt{K\left(\Omega_{2}\right)}\right)^{2}\right\}
$$

and it is an improved version of a similar result from [5]. Substituting $K(\Omega)=2+2 \Gamma(\Omega)$ into the estimation (14) we obtain

$$
K(\Omega) \leq \frac{\left|\Omega_{1}\right|}{\left|\Omega_{s}\right|} K\left(\Omega_{1}\right)+\frac{\left|\Omega_{2}\right|}{\left|\Omega_{s}\right|} K\left(\Omega_{2}\right)-\frac{2|\Omega|}{\left|\Omega_{s}\right|}
$$

which is equivalent with the main result (14) and on the other hand it can be compared to (20). For the sake of simplicity suppose that the plane domain $\Omega_{1}$ is similar to $\Omega_{2}$ and let be $\left|\Omega_{1}\right| \leq\left|\Omega_{2}\right|$. In this case we have $K\left(\Omega_{1}\right)=K\left(\Omega_{2}\right)$ and the minimum in (20) is attained for $j=1$ :

$$
K(\Omega) \leq K\left(\Omega_{1}\right)+\frac{\left|\Omega_{1}\right|}{\left|\Omega_{s}\right|}\left(\sqrt{K\left(\Omega_{1}\right)}+\sqrt{K\left(\Omega_{2}\right)}\right)^{2} .
$$

If $|\Omega| \leq 4 \mid \Omega_{1}$ or if $4\left|\Omega_{1}\right|<|\Omega| \leq 8\left|\Omega_{1}\right|$ and $K\left(\Omega_{1}\right) \leq \frac{2|\Omega|}{|\Omega|-4\left|\Omega_{1}\right|}$, then (21) constitutes a better upper estimation than (22). If $|\Omega|>8\left|\Omega_{1}\right|$, then (22) is definitely better than (21).

Finally we compare Theorem 3.3 to a direct upper estimation for the Velte constant derived in [11] for the class of three-dimensional star-shaped domains. We realize this comparison on an example, where the domain $\Omega$ is the union of two overlapping unit spheres, which is star-shaped for example with respect to the center of the line segment connecting the centers of the spheres. The evaluation of the estimation from [11] yields

$$
\Gamma(\Omega) \leq \frac{2}{9}\left(\frac{2+d}{2-d}\right)^{\frac{3}{2}}\left(\frac{d}{\sqrt{4-d^{2}}}+\sqrt{9+\frac{9 d}{\sqrt{4-d^{2}}}+\frac{d^{2}}{4-d^{2}}}\right)^{2},
$$

where $0<d<2$ denotes the distance of the centers of the spheres. Theorem 3.3 can also be utilized in this case to obtain the upper estimation

$$
\Gamma(\Omega) \leq \frac{64}{(2-d)^{2}(4+d)},
$$

where we have also used that the Velte constant of a sphere equals 2, see [15]. A numerical comparison shows that (24) gives a better upper estimation than (23) if $0,5012 \ldots<d<2$. An even better upper estimation than (24) gives (19) for every $0<d<2$.

\section{Concluding remarks}

In this paper the Friedrichs-Velte and related constants of the union of overlapping domains were investigated. These domain specific constants are especially of interest since they are involved in many problems in fluid dynamics and in elasticity theory but their exact value is known only for a few types of domains. The main result is an upper estimation of the Friedrichs-Velte constant of the union of two overlapping domains in terms of the constants and sizes of the parts and of the size of their intersection. It can be utilized to obtain useful estimations for the discussed constants of more complicated domains using the exact values or upper estimations of the simpler subdomains. It is compared to existing estimations for the related Babuška-Aziz and Korn constant. In order to make this comparison possible we 
developed a new upper estimation for the Babuška-Aziz constant of the union of two overlapping domains based on an existing construction from [8]. A detailed comparison shows that the main result is comparable to existing related ones: it yields occasionally a better upper estimation but it is not definitely better in all cases.

\section{References}

[1] Babuška I., Aziz A.K., Survey lectures on the mathematical foundations of the finite element method, in The mathematical foundations of the finite element method with applications to partial differential equations (Proc. Sympos., Univ. Maryland, Baltimore, Md., 1972), Academic Press, New York, 1972, pp. $1-359$.

[2] Costabel M., Dauge M., On the inequalities of Babuška-Aziz, Friedrichs and Horgan-Payne, Arch. Rational Mech. and Anal. 217 (2015) No 3, 873-898. http://dx.doi.org/10.1007/s00205-015-0845-2

[3] Dauge M., Bernardi C., Costabel M., Girault V., On Friedrichs constant and Horgan-Payne angle for LBB condition, Monografiás Matemáticas García de Galdeano 39, 87-100 (2014).

[4] Costabel M.: Inequalities of Babuška-Aziz and Friedrichs-Velte for differential forms, HAL: hal01181963, arXiv:1507.08464 (2015).

[5] Dafermos C. M., Some Remarks on Korn's Inequality. Zeitschrift für Angewandte Mathematik und Physik 19 (1968), 913-920. http://dx.doi.org/10.1007/BF01602271

[6] Durán R.G., Muschietti M.A., The Korn inequality for Jones domains, Electronic Journal of Differential Equations, Vol. 2004 (2004), No. 127, 1-10.

[7] Friedrichs K., On certain inequalities and characteristic value problems for analytic functions and for functions of two variables. Trans. AMS 41 (1937), 321-364.

[8] Galdi, G. P.: An Introduction to the Mathematical Theory of the Navier-Stokes Equations: Linearized Steady Problems, Band 1., Springer-Verlag (1994). http://dx.doi.org/10.1007/978-1-4757-3866-7

[9] Horgan C.O. and Payne L.E., On inequalities of Korn, Friedrichs and Babuška-Aziz. Achive Rat. Mech. Anal. 82 (1983), 165-179. http://dx.doi.org/10.1007/BF00250935

[10] Kessler M., Die Ladyzhenskaya-Konstante in der numerischen Behandlung von Strömungsproblemen, Thesis, Bayerische Julius-Maximilians-Universität, Würzburg (2000).

[11] Payne L.E., A bound for the optimal constant in an inequality of Ladyzhenskaya and Solonnikov, IMA Journal of Applied Mathematics 72 (2007), 563-569. http://dx.doi.org/10.1093/imamat/hxm028

[12] Shapiro H.S., On some Fourier and distribution-theoretic methods in approximation theory, in vol. Approximation Theory. III, Proc. Conf. held in Austin, Texas, 1980 (W.Cheney et. al., eds.), Academic Press, San Diego (1980), 87-124.

[13] Stoyan G., Towards discrete Velte decompositions and narrow bounds for inf-sup constants, Computers \& Maths. with Appls., 38, 7-8, (1999), 243-261. http://dx.doi.org/10.1016/S0898-1221(99)00254-0

[14] Stoyan G., Iterative Stokes solvers in the harmonic Velte subspace, Computing 67 (2000), 13-33. http://dx.doi.org/10.1007/s006070170014

[15] Velte W., On inequalities of Friedrichs and Babuška-Aziz in dimension three, Journal for Analysis and its Applications, Vol.17, No.4 (1998), 843-857. http://dx.doi.org/10.4171/ZAA/854

[16] Zsuppán S., On the domain dependence of the inf-sup and related constants via conformal mapping, J. Math. Anal. Appl. 382 (2011), 856-863. http://dx.doi.org/10.1016/j.jmaa.2011.04.086

[17] Zsuppán S., On the spectrum of the Schur complement of the Stokes operator via conformal mapping, Methods and Applications of Analysis, Vol.11, No.1 (2004), 133-154. http://dx.doi.org/10.4310/MAA.2004.v11.n1.a8

[18] Zsuppan S., On the Stokes problem, Thesis, Eötvös Loránd Univ. Budapest, 2008. 